\title{
Prediction of Postoperative Mortality in Patients with Organ Failure After Gastric Cancer Surgery
}

\author{
Ji-Ho Park ${ }^{1,2}$ (1) Hyuk-Joon Lee ${ }^{1,3} \cdot$ Seung-Young Oh ${ }^{1,4} \cdot$ Shin-Hoo Park $^{1} \cdot$ Felix Berlth $^{1,5}$. \\ Young-Gil Son ${ }^{1,6} \cdot$ Tae Han Kim ${ }^{1,2} \cdot$ Yeon-Ju Huh ${ }^{1,7} \cdot$ Jun-Young Yang ${ }^{1,8} \cdot$ Kyung-Goo Lee $^{1,9}$ • \\ Yun-Suhk Suh ${ }^{1} \cdot$ Seong-Ho Kong ${ }^{1} \cdot$ Han-Kwang Yang ${ }^{1,3}$
}

Published online: 28 January 2020

(C) Société Internationale de Chirurgie 2020

\begin{abstract}
Background Scarce data are available on the characteristics of postoperative organ failure (POF) and mortality after gastrectomy. We aimed to describe the causes of organ failure and mortality related to gastrectomy for gastric cancer and to identify patients with POF who are at a risk of failure to rescue (FTR).

Methods The study examined patients with POF or in-hospital mortality in Seoul National University Hospital between 2005 and 2014. We identified patients at a high risk of FTR by analyzing laboratory findings, complication data, intensive care unit records, and risk scoring including Acute Physiology and Chronic Health Evaluation (APACHE) IV, Sequential Organ Failure Assessment (SOFA) score, and Simplified Acute Physiology Score (SAPS) 3 at ICU admission.

Results Among the 7304 patients who underwent gastrectomy, 80 (1.1\%) were identified with Clavien-Dindo classification (CDC) grade $\geq \mathrm{IVa}$. The numbers of patients with CDC grade IVa, IVb, and V were $48(0.66 \%), 11$ $(0.15 \%)$, and $21(0.29 \%)$, respectively. Pulmonary failure (43.8\%), surgical site complication $(27.5 \%)$, and cardiac failure (13.8\%) were the most common causes of POF and mortality. Cancer progression (100\%) and cardiac events $(45.5 \%)$ showed high FTR rates. In univariate analysis, acidosis, hypoalbuminemia, SOFA, APACHE IV, and SAPS 3 were identified as risk factors for FTR $(P<0.05)$. Finally, SAPS 3 was identified as an independent predictive factor for FTR.

Conclusions Cancer progression and acute cardiac failure were the most lethal causes of FTR. SAPS 3 is an independent predictor of FTR among POF patients after gastrectomy.
\end{abstract}

Hyuk-Joon Lee

appe98@snu.ac.kr

1 Department of Surgery, Seoul National University College of Medicine, Seoul, South Korea

2 Department of Surgery, Gyeongsang National University Hospital, Jinju, South Korea

3 Department of Surgery, Cancer Research Institute, Seoul National University College of Medicine, Seoul, South Korea

4 Critical Care Center, Seoul National University College of Medicine, Seoul, South Korea
Department of General, Visceral and Cancer Surgery, University Hospital of Cologne, Cologne, Germany

6 Department of Surgery, Keimyung University School of Medicine, Daegu, South Korea

7 Department of Surgery, Ewha Womans University Mokdong Hospital, Seoul, South Korea

8 Department of Surgery, Gachon University Gil Medical Center, Incheon, South Korea

9 Department of Surgery, Myongji Hospital, Goyang, South Korea 


\section{Introduction}

Gastrectomy is the mainstay treatment for patients with gastric cancer. The prediction and management of postoperative morbidity become more important as a trend is seen recently in older and more fragile patients $[1,2]$. Despite significant efforts to reduce complications, the morbidity rate following gastrectomy is reported to be $10-28 \%$ [3-9].

Among postoperative complications of gastric cancer surgery, organ failure and in-hospital mortality after gastrectomy are rare. Based on the Clavien-Dindo classification (CDC) [10], grade IV is defined as life-threatening complications requiring intensive care unit (ICU) management. In addition, grade IVa is defined as single organ dysfunction requiring dialysis, or mechanical ventilation, and grade IVb refers to multiple organ failure. Grade V is defined as the complication-related death of patients. In previous reports, the rates of grade IV and $\mathrm{V}$ complications were $0.6-1.1 \%$ and $0.1-0.5 \%$, respectively $[6,11]$. In most other studies addressing postoperative complications after gastrectomy for gastric cancer, grade IV complication rates have not been specifically mentioned [3-5]. Reasons for lack of data might cause difficulty in clearly defining the CDC grade IV complications, complexity of ICU treatments, and vast number of other parameters that influence the appropriate classification.

Failure to rescue (FTR) patients with complications account for a substantial proportion of postoperative mortality and is an emerging indicator of the quality of medical care [12, 13]. Understanding the factors responsible for FTR helps surgeons predict and avoid operative mortality. However, there are very few studies related to FTR in the field of gastric cancer surgery [14].

Hence, in this study, we described the causes of organ failure and mortality related to gastrectomy for gastric cancer and identified patients with postoperative organ failure who are at a risk of FTR.

\section{Materials and methods}

\section{Patient population and data source}

We screened the Seoul National University Hospital gastric cancer database from 2005 to 2014 and identified patients with postoperative organ failure and in-hospital mortality. A total of 7304 patients underwent gastrectomy for histologically confirmed gastric cancer within the study period. Among these patients, we collected data for patients meeting the following inclusion criteria: 30-day and inhospital mortality (CDC grade V); transfer to ICU because of organ failure (CDC grade IV); and continuous postoperative ICU care for 3 or more days and occurrence of any organ failure (CDC grade IV). Patients who underwent palliative procedures without gastrectomy (e.g., gastro-jejunostomy and explorative laparotomy) or procedures in cooperation with other surgical techniques (e.g., coronary artery bypass graft and abdominal aorta aneurysm operation) were excluded. Patients who underwent combined organ resection (e.g., colon resection, cholecystectomy, and pancreaticoduodenectomy) owing to gastric cancer were included in our analysis.

Demographics, comorbidity, operative procedures, pathological results based on the American Joint Committee on Cancer (AJCC) TNM 7th edition [15], ICU medical record, laboratory data, and postoperative complications were reviewed. Preoperative comorbidity data were collected and graded using the Charlson comorbidity index, which is the most widely used method to determine the overall burden of comorbidities and includes 19 medical conditions with corresponding weights [16]. Complications data based on CDC were collected prospectively through weekly team meetings [10]. The type of complications was divided into two categories: local and systemic. Complications were defined and grouped based on our previous report on complication following gastrectomy [11]. We calculated the time to the development of the first complication as the number of days between the operation and the first documented postoperative complication. This study was approved by the Institutional Review Board of our institution (H-1305-043-488).

\section{Assignment of causes of organ failure and mortality}

The causes of organ failure and mortality were systematically described by applying the methodology proposed by Waljee et al. [17]. In this classification, the complication that most commonly contributed to patient's ICU transfer or death was defined as a "seminal" complication. We adopted this concept when determining the cause of organ failure and mortality. Based on this classification and clinical relevance and frequency of occurrence, we allocated the cases based on the causes of organ failure and mortality into six categories with slight modifications: pulmonary failure (pneumonia, prolonged ventilator assistance, pulmonary edema, pulmonary thromboembolism, pneumothorax); surgical site complications (hemorrhage and surgical site infection); cardiac complication (myocardial infarction, congestive heart failure, arrhythmia, and unexplained cardiac arrest); cerebrovascular complications (stroke); cancer progression; and other complications (urinary tract infection and suicide). Three attending surgeons (HJ Lee, JH Park, and SY Oh) independently reviewed all medical records including ICU data and 
Table 1 Variables included in the prognostic models

\begin{tabular}{|c|c|c|c|}
\hline Predictor variable & SAPS 3 & APACHE IV & SOFA \\
\hline Age & + & + & - \\
\hline Length of hospital stay before ICU admission & + & + & - \\
\hline ICU admission source (number of items) & 3 & 8 & - \\
\hline Chronic comorbidities (number of items) & 6 & 7 & - \\
\hline Surgical status at ICU admission & + & + & - \\
\hline Anatomic site of surgery & + & - & - \\
\hline Reasons for ICU admission/acute diagnosis (number of items) & 10 & 116 & - \\
\hline Acute infection at ICU admission & + & - & - \\
\hline Mechanical ventilation & + & + & + \\
\hline Vasoactive drug therapy & + & - & + \\
\hline Clinical physiological variables (number of items) & 4 & 6 & 3 \\
\hline Laboratory physiological variables (number of items) & 6 & 10 & 5 \\
\hline
\end{tabular}

SAPS Simplified Acute Physiology Score; APACHE Acute Physiology and Chronic Health Evaluation; SOFA Sequential Organ Failure Assessment; ICU intensive care unit

determined the cause of organ failure and death. In case of disagreement, the medical record was reviewed by the entire committee. After discussion, a final consensus on the cause of organ failure and death was reached.

\section{Outcomes for risk analysis of failure to rescue}

To identify risk factors for FTR, risk scoring including the Charlson comorbidity index, Acute Physiology and Chronic Health Evaluation (APACHE) IV, Sequential Organ Failure Assessment (SOFA; formerly, Sepsis-Related Organ Failure Assessment) score, and SAPS (Simplified Acute Physiology Score) 3; laboratory findings; complication details; and ICU clinical data were reviewed. All clinical and laboratory data references for risk scoring were collected within the initial $24 \mathrm{~h}$ after ICU admission. Two patients who died suddenly without ICU care and one patient who committed suicide were excluded from FTR risk analysis.

APACHE IV is a widely used scoring system for assessing the severity of illness and prognosis of ICU patients [18]. This scoring system includes age, chronic health conditions, and physiologic data required to calculate an acute physiology score of APACHE III [19]. This score also involves the primary reason for ICU admission (ICU admission diagnosis), patient's location (admission source), length of stay before ICU admission, need of mechanical ventilation or emergency surgery, $\mathrm{PaO}_{2} / \mathrm{FiO}_{2}$ ratio, and whether sedation or paralysis resulted in an inability to assess the Glasgow Coma Scale (GCS).

SAPS 3 is a model for predicting mortality and is one of the several ICU scoring systems. It was designed to provide a real-life prediction of mortality for patients. The model is based on a mathematical model that needs calibration [20].
SAPS 3 represents the arithmetic sum of three boxes: (1) Box I: age, comorbidities, use of vasoactive drugs before ICU admission, intrahospital location before ICU admission, and length of stay in the hospital before ICU admission; (2) Box II: reasons for ICU admission, planned/ unplanned ICU admission, surgical status at ICU admission, anatomical site of surgery, and presence of infection at ICU admission; and (3) Box III: lowest estimated GCS, highest heart rate, lowest systolic blood pressure, highest bilirubin, highest body temperature, highest creatinine, highest leukocytes, lowest platelets, lowest hydrogen ion concentration $(\mathrm{pH})$, and ventilatory support and oxygenation.

The SOFA score is used to track a patient's status during the stay in an ICU in order to determine the extent of a patient's organ function or rate of failure [21]. The score is based on six different sub-scores, one each for the respiratory, cardiovascular, hepatic, coagulation, renal, and neurological systems.

These scoring data were collected for the first $24 \mathrm{~h}$ of admission to the ICU and were entered into a computerbased calculator (APACHE IV, https://intensivecarenet work.com/Calculators/Files/Apache4.html; SAPS 3, http:// www.saps3.org/resources-downloads/user-agreement/ downloads/; and SOFA, https://www.mdcalc.com/sequen tial-organ-failure-assessment-sofa-score). A comparison of the three scores is shown in Table 1.

\section{Statistical analysis}

The categorical variables were presented as numbers and percentages, and the groups by FTR were compared using the chi-square test. Continuous variables were expressed as means and standard deviations, and the means were 
Table 2 Patients' characteristics

\begin{tabular}{|c|c|}
\hline Variable & Total $(N=80)$ \\
\hline Age & $67.3(35-83)$ \\
\hline Sex ratio $(M / F)$ & $67: 13$ \\
\hline Comorbidity & $67(83.8 \%)$ \\
\hline ASA score (I:II:III) & $11: 55: 13$ \\
\hline \multicolumn{2}{|l|}{ Complication grade ${ }^{\mathrm{a}}$} \\
\hline IVa & $48(60.0 \%)$ \\
\hline $\mathrm{IVb}$ & $11(13.8 \%)$ \\
\hline $\mathrm{V}$ & $21(26.3 \%)$ \\
\hline \multicolumn{2}{|l|}{ Extent of gastrectomy } \\
\hline Distal gastrectomy & $39(48.8 \%)$ \\
\hline Pylorus-preserving gastrectomy & $4(5.0 \%)$ \\
\hline Proximal gastrectomy & $3(3.8 \%)$ \\
\hline Total gastrectomy & $34(42.5 \%)$ \\
\hline \multicolumn{2}{|l|}{ Surgical method } \\
\hline Open & $66(82.5 \%)$ \\
\hline Laparoscopic or robotic & $14(17.5 \%)$ \\
\hline \multicolumn{2}{|l|}{ Lymph node dissection } \\
\hline D1 or D1+ & $20(25.0 \%)$ \\
\hline D2 or more & $60(75.0 \%)$ \\
\hline \multicolumn{2}{|l|}{ Combined resection } \\
\hline No & $55(68.8 \%)$ \\
\hline Yes & $25(31.3 \%)$ \\
\hline \multicolumn{2}{|l|}{ Surgical radicality } \\
\hline R0 & $67(83.8 \%)$ \\
\hline $\mathrm{R} 1$ or $\mathrm{R} 2$ & $13(16.2 \%)$ \\
\hline \multicolumn{2}{|l|}{ Tumor invasion } \\
\hline EGC & $30(37.5 \%)$ \\
\hline AGC & $50(62.5 \%)$ \\
\hline \multicolumn{2}{|l|}{ Lymph node metastasis } \\
\hline Negative & $37(46.3 \%)$ \\
\hline Positive & $43(53.7 \%)$ \\
\hline \multicolumn{2}{|l|}{ TNM stage } \\
\hline I & $36(45.0 \%)$ \\
\hline II & $7(8.8 \%)$ \\
\hline III & $26(32.5 \%)$ \\
\hline IV & $11(13.8 \%)$ \\
\hline
\end{tabular}

ASA American Society of Anesthesiologists; EGC early gastric cancer; $A G C$ advanced gastric cancer

${ }^{a}$ According to the Clavien-Dindo classification

compared using the Student's $t$ test. Univariate analysis was conducted to evaluate the association of each factor with FTR. The identified risk factors for FTR were adjusted by multivariate logistic regression. For this purpose, significant variables $(P<0.05)$ in the univariate analysis were entered into the multivariate analysis. In multivariate analysis, the adjusted odds ratios (exponential beta) were calculated with a $95 \%$ confidence interval (CI). All tests were two-sided and performed at a significance level of 5\% using IBM $^{\circledR}$ SPSS $^{\circledR}$ Statistics version 20 (IBM Corporation, Armonk, NY, USA).

Results

\section{Patient characteristics, comorbidities, and complications}

Among the 7304 patients who underwent gastrectomy, 80 $(1.1 \%)$ were identified with postoperative organ failure and/or mortality (CDC grade $\geq$ IVa). The numbers of patients with CDC grade IVa, IVb, and V complications were $48(0.66 \%), 11(0.15 \%)$, and $21(0.29 \%)$, respectively. All 80 patients were examined, and their clinicopathological characteristics are shown in Table 2. The study cohort included 67 men and 13 women, with a mean age of 67.3 (35-83) years. Eighty-three percent (67/80) of the patients had preoperative medical comorbidities. Additionally, $85 \%$ of the patients received an American Society of Anesthesiologists (ASA) score of 2 or higher (ASA I:II:III = 11:55:13). In terms of tumor invasion, advanced gastric cancer occurred more frequently than did early gastric cancer, and more than half of the patients $(53.7 \%, 43 / 80)$ had lymph node metastasis. The distribution of TNM stage I, II, III, and IV was found to be 36 (45\%), 7 (8.8\%), $26(32.5 \%)$, and 11 (13.8\%), respectively.

Comorbidities and complication details are shown in Table 3. Hypertension (52.5\%), diabetes mellitus (27.5\%), heart disease $(18.8 \%)$, lung disease $(17.5 \%)$, and neurological diseases $(15 \%)$ were common comorbid conditions. Detailed complications were grouped as local and systemic complications. All patients had systemic complications. Local complications developed in about half of the patients (52.5\%, 42/80). Pulmonary (86.3\%), cardiac (38.8\%), and renal complication (25\%), fluid collection (23.8\%), bleeding $(22.5 \%)$, and operation site leakage $(20 \%)$ were the common complications seen.

\section{Causes of organ failure and mortality}

Table 4 shows the detailed causes of organ failure and ICU admission of the 80 patients included in this study. The most common causes of organ failure were respiratory failure $(35,43.8 \%)$ and surgical site complications (22, $27.5 \%)$, followed by cardiac events $(11,13.8 \%)$, cerebrovascular complications $(6,7.5 \%)$, cancer progression $(4$, $5 \%$ ), and other complications $(2,2.5 \%)$. The most common seminal complications were pneumonia $(24,30 \%)$ and surgical site infection $(13,16.3 \%)$, such as bowel leakage and intra-abdominal abscess, followed by hemorrhage ( 9 , $11.3 \%)$, stroke $(6,7.5 \%)$, acute myocardial infarction (5, 
Table 3 Comorbidity and complication details

\begin{tabular}{lc}
\hline Variable & Total $(N=80)$ \\
\hline Comorbidity & $67(83.8 \%)$ \\
Hypertension & $42(52.5 \%)$ \\
Diabetes & $22(27.5 \%)$ \\
Tuberculosis & $12(15.0 \%)$ \\
Chronic liver disease & $8(10.0 \%)$ \\
Pulmonary disease & $14(17.5 \%)$ \\
Cardiac disease & $15(18.8 \%)$ \\
Neurologic disease & $12(15.0 \%)$ \\
Renal disease & $9(11.3 \%)$ \\
Other malignancy & $6(7.5 \%)$ \\
Other disease & $3(3.8 \%)$ \\
Local complication & $42(52.5 \%)$ \\
Wound problem & $11(13.8 \%)$ \\
Fluid collection & $19(23.8 \%)$ \\
Intra-abdominal bleeding & $12(15 \%)$ \\
Intra-luminal bleeding & $6(7.5 \%)$ \\
Stenosis & $1(1.3 \%)$ \\
Ileus/motility disorder & $11(13.8 \%)$ \\
Anastomotic leakage & $16(20 \%)$ \\
Other leakage/fistula & $4(5 \%)$ \\
Vascular insufficiency & $2(2.5 \%)$ \\
Systemic complication & $80(100 \%)$ \\
Pulmonary & $69(86.3 \%)$ \\
Urinary & $5(6.3 \%)$ \\
Renal & $20(25.0 \%)$ \\
Hepato-biliary & $9(11.3 \%)$ \\
Cardiac & $31(38.8 \%)$ \\
Endocrine & $1(1.3 \%)$ \\
Neurologic & $5(21.3 \%)$ \\
Vascular & $1(6.3 \%)$ \\
Others ${ }^{a}$ &
\end{tabular}

${ }^{\mathrm{a}}$ One patient died of suicide

$6.3 \%)$, prolonged ventilator assistance $(4,5 \%)$, pulmonary edema $(4,5 \%)$, and cancer dissemination $(4,5 \%)$. One patient $(1.3 \%)$ committed suicide in the ward 3 days after surgery.

The grouped cause of organ failure affects the FTR rate (Fig. 1). Cancer progression had the highest mortality rate $(100 \%, 4 / 4)$. Cardiac events $(45.5 \%, 5 / 11)$ and surgical site complication $(31.8 \%, 7 / 22)$ showed a relatively high FTR rate. However, the lowest mortality rate was observed with pulmonary complications $(8.6 \%, 3 / 35)$.

\section{Clinical data related to failure to rescue}

In univariate analysis (Table 5), low albumin level and acidosis were significantly different between patients with
Table 4 Classification of the cause of organ failure

\begin{tabular}{llc}
\hline Cause of organ failure & Seminal complication & $\mathrm{N}=80$ \\
\hline Pulmonary failure & Pneumonia & $24(30.0 \%)$ \\
& $\begin{array}{l}\text { Prolonged ventilator } \\
\text { assistance }\end{array}$ & $4(5.0 \%)$ \\
& Pulmonary edema & $4(5.0 \%)$ \\
& Pulmonary & $2(2.5 \%)$ \\
& thromboembolism & \\
Surgical site complication & Peumothorax & $1(1.5 \%)$ \\
& Surgical site infection & $13(16.3 \%)$ \\
Cardiac complication & Myocardial infarction & $5(6.3 \%)$ \\
& Congestive heart failure & $2(2.5 \%)$ \\
& Arrhythmia & $2(2.5 \%)$ \\
Cerebral vascular & Unexplained cardiac arrest & $2(2.5 \%)$ \\
complication & Stroke & $6(7.5 \%)$ \\
Cancer progression & Cancer dissemination & $4(5.0 \%)$ \\
Others & Urinary tract infection & $1(1.3 \%)$ \\
& Suicide & $1(1.3 \%)$ \\
\hline
\end{tabular}

rescue and FTR $(P=0.026$ and $P=0.001$, respectively $)$ at initial ICU laboratory findings. In terms of risk scoring methods, APACHE IV, SOFA, and SAPS 3 were significantly associated with the FTR rate $(P=0.001, P<0.001$, and $P<0.001$, respectively). Complication-related data, including type, timing, and time, to the development of the organ failure were not related to FTR.

In multivariate analysis, SAPS 3 was found to be the only independent predictor of FTR (OR 1.09, 95\% CI 1.033-1.149; $P=0.002$ ) (Table 6).

\section{Discussion}

To our knowledge, this is one of the first reports addressing the causes of organ failure and mortality in patients undergoing gastrectomy for gastric cancer. In this context, we conducted a cohort-based study using large-volume data to be able to describe the causes of organ failure and mortality related to gastrectomy. In addition to comparing outcomes between the rescued and FTR patients, we assessed the risks for in-hospital death. Severe complications (CDC grade $\geq$ IV) developed in only $1.1 \%$ of the patients. The characteristics of these patients were heterogenous in terms of demographics, underlying diseases, and complications. The main causes of organ failure and death were pulmonary failure and surgical site complications that accounted for more than $70 \%$. On the other hand, in relation to the grouped number of patients with organ failure, pulmonary failure had the lowest mortality 
Fig. 1 Morality rate according to cause of organ failure

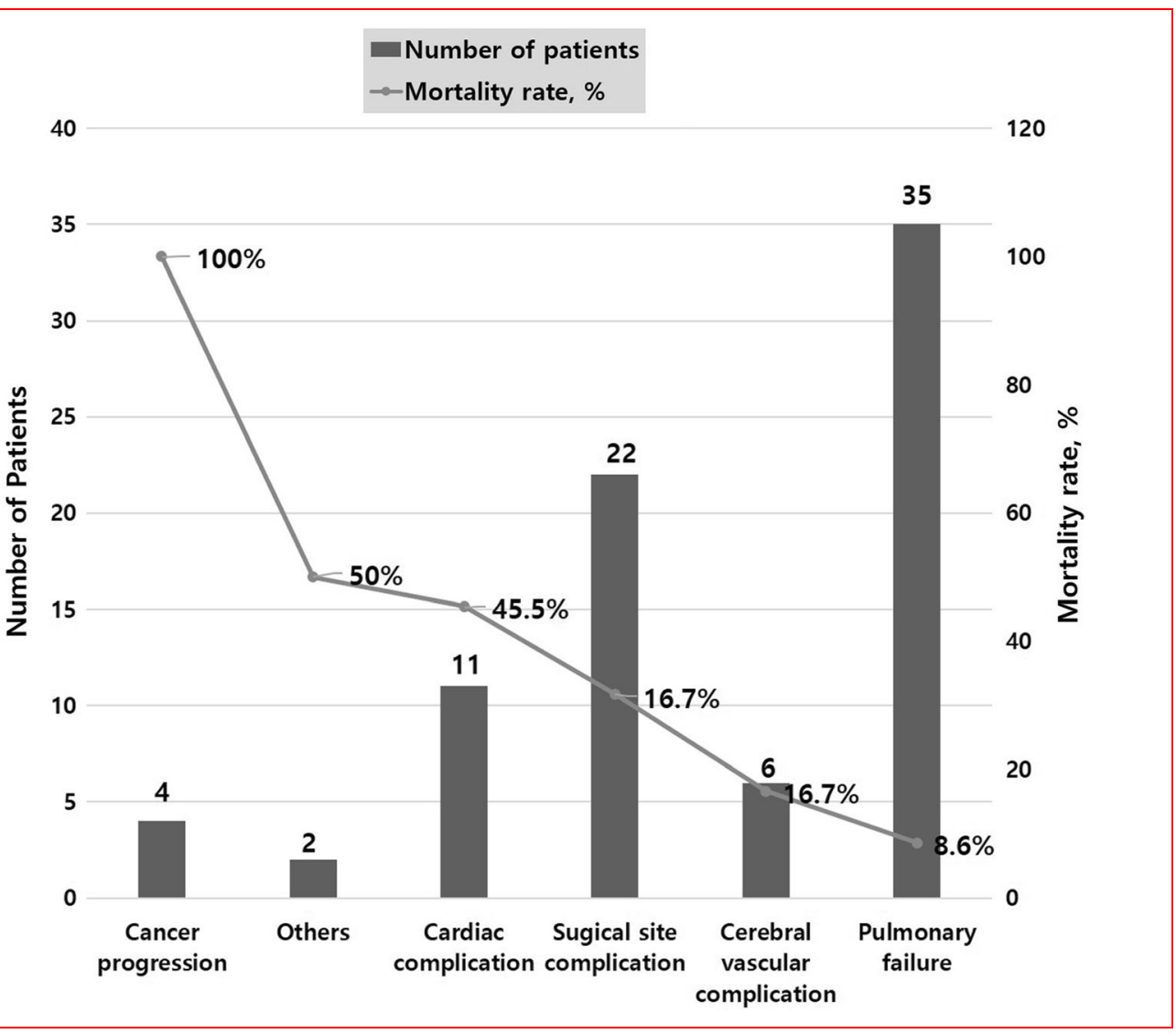

Table 5 Clinical and laboratory data at intensive care unit related to failure to rescue

\begin{tabular}{|c|c|c|c|}
\hline Variable & Rescue $(N=59)$ & FTR $(N=18)$ & $P$ \\
\hline Age & $68.0 \pm 10.0$ & $66.9 \pm 11.5$ & 0.688 \\
\hline Charlson comorbidity score & $3.1 \pm 1.1$ & $3.7 \pm 1.5$ & 0.144 \\
\hline Platelet $\left(\times 10^{3} / \mu \mathrm{L}\right)$ & $181.8 \pm 78.3$ & $187.6 \pm 143.7$ & 0.871 \\
\hline Bilirubin (mg/dL) & $1.6 \pm 0.9$ & $4.2 \pm 5.9$ & 0.076 \\
\hline Albumin $(\mathrm{g} / \mathrm{dL})$ & $2.6 \pm 0.4$ & $2.4 \pm 0.4$ & 0.026 \\
\hline Creatinine $(\mathrm{mg} / \mathrm{dL})$ & $1.4 \pm 1.4$ & $1.6 \pm 0.9$ & 0.645 \\
\hline $\mathrm{pH}$ & $7.36 \pm 0.08$ & $7.27 \pm 0.12$ & 0.001 \\
\hline APACHE IV & $69.7 \pm 25.7$ & $96.1 \pm 39.6$ & 0.001 \\
\hline SOFA & $7.2 \pm 3.0$ & $11.1 \pm 3.3$ & $<0.001$ \\
\hline SAPS 3 & $58.6 \pm 14.0$ & $79.2 \pm 15.0$ & $<0.001$ \\
\hline Type of 1 st complication (local/systemic) & 19:40 & $10: 8$ & 0.092 \\
\hline Postoperative day of 1st complication & $3.9 \pm 4.5$ & $5.8 \pm 6.7$ & 0.250 \\
\hline Postoperative day of ICU admission & $4.1 \pm 5.0$ & $25.0 \pm 60.9$ & 0.161 \\
\hline Day from 1st complication to ICU admission & $0.6 \pm 1.5$ & $19.8 \pm 58.6$ & 0.183 \\
\hline
\end{tabular}

FTR failure to rescue; APACHE Acute Physiology and Chronic Health Evaluation; SOFA Sequential Organ Failure Assessment; SAPS Simplified Acute Physiology Score; ICU intensive care unit

rate among the five groups. SAPS 3 risk scoring was the most powerful predictor for FTR in ICU patients.

The mortality rate after gastrectomy in this study was $0.29 \%$. The literature reports operative mortality rates that range from 0 to $13 \%$, with higher mortality rates in
Western studies (the least reported at 4\%) [3-5, 8, 9, 14, 22-25]. The most recently published East Asian, multicenter, randomized controlled trials have reported a very low mortality rate of less than $0.5 \%$ $[3,24,25]$, which was similar to that seen in our study. 
Table 6 Predictive factors for failure to rescue by multivariate analysis

\begin{tabular}{llll}
\hline Variable & $P$ & $\begin{array}{l}\text { Exponential } \\
(ß)\end{array}$ & $95 \%$ CI \\
\hline Albumin (g/dL) & 0.354 & & \\
$\mathrm{pH}$ & 0.177 & & \\
APACHE IV & 0.270 & & \\
SOFA & 0.257 & & $1.033-1.149$ \\
SAPS 3 & 0.002 & 1.090 & \\
Type of 1st complication & 0.652 & & \\
$\quad$ local/systemic) & & & \\
\hline
\end{tabular}

APACHE Acute Physiology and Chronic Health Evaluation; SOFA Sequential Organ Failure Assessment; SAPS Simplified Acute Physiology Score

However, our retrospective analysis included all gastric cancer patients who underwent gastrectomy unlike randomized controlled trials with strict inclusion and exclusion criteria.

Organ failure is organ dysfunction to such a degree that homeostasis cannot be maintained without external clinical intervention. If it involved two or more organ systems, it is defined as multiple organ failure, which is the most common cause of death in the surgical ICU [26]. Most studies on postsurgical morbidity focused on major complications (more than CDC grade III) and mortality. However, there was no analysis of specific details published with a focus on organ failure (grade IV) with or without consecutive mortality (grade V) after gastrectomy for gastric cancer. In this study, we investigated the characteristics of patients with organ failure after gastrectomy. Compared to our previous two studies that dealt with the association of underlying diseases and complications after gastrectomy $[27,28]$, we found a higher prevalence of comorbidities $(83.8 \%, 67 / 80)$ that was associated with the development of organ failure. In particular, the proportion of hepatic, pulmonary, cardiac, renal, and neurological underlying diseases were highly related to the patients' vitality. Among the complications, all patients had systemic complications, and the rate of surgical complications such as bleeding or leakage was also high.

To our knowledge, there is no study that classifies the causes of organ failure after surgery. Therefore, we introduced the methodology proposed by Waljee et al. [17] for classifying the cause of death after cancer surgery. Gerestein et al. [29] reported the causes of postoperative mortality after surgery for ovarian cancer. Surgical site complications and pulmonary failure were common causes of mortality in that study. In our study, organ failure was caused by pulmonary failure, surgical site complication, cardiac complication, and cerebrovascular complications, sequentially. Whereas pulmonary failure had a mortality of
$8.6 \%$, mortality rate according to the cause of organ failure was the highest in cancer progression $(100 \%)$, followed by cardiac complications and surgical site infections. These results can be mainly explained by sudden cardiac death, myocardial infarction, and sepsis caused by surgical site infection. In particular, surgical site infections influenced organ failure included bowel leakage, intraperitoneal abscess, bile leakage, and gallbladder abscess.

Although several reports identified patients' factors predicting postoperative death, few reports investigated the risk for FTR among patients who were admitted to the ICU with organ failure. Bartlett et al. [30] investigated specific patient factors associated with mortality after total gastrectomy using the American College of Surgeons National Surgical Quality Improvement Program database. Age $>70$ years, weight loss, albumin $<3 \mathrm{~g} / \mathrm{dL}$, and combined resection of the pancreas were independently associated with increased 30-day mortality. Another American study showed that patient characteristics, including age and comorbidity, were highly significant predictors of mortality [31]. Recently, Japanese surgeons have reported a risk stratification study for gastrectomy when treating gastric cancer using a nationwide Web-based database $[8,9]$. This risk models included age, need for total assistance in activities of daily living, American Society of Anesthesiologists grade 4 or 5 , disseminated cancer, preoperative dialysis requirement, cerebrovascular disease history, more than $10 \%$ weight loss, uncontrolled ascites, leukocytosis, thrombocytopenia, anemia, hypoalbuminemia, hyponatremia, and elevated alkaline phosphatase level.

In our study, hypoalbuminemia and acidosis were remarkable factors for FTR. Additionally, risk scoring using APACHE IV, SOFA, and SAPS 3 was significantly associated with mortality. Finally, SAPS 3 was identified as an independent predictive factor for FTR among patients with organ failure after gastrectomy. In the acute care surgical field, characteristics of complications such as type of seminal complication and number of complications were reported as significant prognostic factors for FTR [32, 33]. However, details about complication (type of 1st complication, duration, timing of complication) did not influence FTR in our study.

Prognostic scoring systems have been developed by the critical care community as an effort to quantify the severity of the illness of a given patient or group of patients [34]. Many prognostic models exist, suggesting that the optimum model has not been established. In particular, few studies have applied risk scoring systems to ICU patients with gastric cancer. The most commonly used adult ICU prognostic scoring systems are APACHE and SAPS. Major revisions of these models were published between 2005 and 2006, namely APACHE IV in 2006 and SAPS 3 in $2005[18,20]$. Moreover, the SOFA score is used to track a 
patient's status during the stay in an ICU to determine the organ function or rate of failure. In this study, we analyzed these three systems and SAPS 3 was most useful in predicting FTR among gastric cancer patients with organ failure. Unlike other systems, SAPS 3 includes data regarding the anatomical site of surgery, presence of acute infection at ICU admission, and vasoactive drug therapy before ICU admission (Table 1). These factors might contribute to the accuracy of the analysis of gastric cancer patients. Future studies are needed to evaluate the role of risk scoring or benchmarking, performance improvement, resource use, and clinical decision support.

According to the SAPS 3 system, the patient prognosis factor can be divided into three categories: (a) previous medical/surgical history, (b) the circumstances of ICU admission, and (c) the degree and presence of physiologic dysfunction, and the prognostic value to resuscitate in each category is $50 \%, 22.5 \%$, and $27.5 \%$, respectively [20]. More than half of the risk for mortality is already determined when a patient is admitted to the ICU. In order to reduce mortality, it is advisable to correct physiologic indices when a high risk of mortality is suspected as soon as possible and perform prompt assessments to identify patient status. In this system, mortality differs in the etiology for ICU admission; transient organ failure shows a better chance of recovery compared to definite conditions such as intracranial mass or severe pancreatitis.

After gastrectomy, organ dysfunction and death are rare events and are therefore difficult to investigate. We performed analyses with mortality and organ failure of patients after gastric cancer surgery through complementary crossing over review using well-organized data from the large-volume gastric cancer center. Prevention of mortality rate is crucial for control of healthcare quality. Our results are intended to contribute to the management of patients with severe complications leading to death after gastric cancer surgery. This is expected to lead to an improvement in the quality of surgery.

In summary, postoperative organ failure and mortality after gastrectomy have heterogeneous causes. Information on morbidities and causes of organ failure or mortality seems essential for managing fatal complications. Cancer progression and acute cardiac failure were the most lethal causes of FTR. SAPS 3 is an independent predictor of FTR among organ failure patients after gastrectomy. By characterizing fatal morbidity and mortality after gastric cancer surgery, it is possible to provide vital information in efforts to reduce hospital mortality.

Acknowledgements This study was supported by a research grant from the Seoul National University Hospital Research Fund (No. 0420133080).
Funding This study was funded by a research grant from the Seoul National University Hospital Research Fund (No. 0420133080).

\section{Compliance with ethical standards}

Conflicts of interest The authors declare that they have no conflict of interest.

\section{References}

1. Park JH, Son YG, Kim TH et al (2017) Identification of candidates for early discharge after gastrectomy. Ann Surg Oncol 24:159-166

2. Yang JY, Lee HJ, Kim TH et al (2017) Short-and long-term outcomes after gastrectomy in elderly gastric cancer patients. Ann Surg Oncol 24:469-477

3. Kim W, Kim HH, Han SU et al (2016) Decreased morbidity of laparoscopic distal gastrectomy compared with open distal gastrectomy for stage I gastric cancer. Ann Surg 263:28-35

4. Sasako M, Sano T, Yamamoto S et al (2008) D2 lymphadenectomy alone or with para-aortic nodal dissection for gastric cancer. N Engl J Med 359:453-462

5. Degiuli M, Sasako M, Ponti A (2010) Morbidity and mortality in the Italian Gastric Cancer Study Group randomized clinical trial of D1 versus D2 resection for gastric cancer. $\mathrm{Br} \mathrm{J}$ Surg 97:643-649

6. Kim TH, Suh YS, Huh YJ et al (2018) The comprehensive complication index (CCI) is a more sensitive complication index than the conventional Clavien-Dindo classification in radical gastric cancer surgery. Gastric Cancer 21:171-181

7. Park DJ, Lee HJ, Kim HH et al (2005) Predictors of operative morbidity and mortality in gastric cancer surgery. Br J Surg 92:1099-1102

8. Kurita N, Miyata H, Gotoh M et al (2015) Risk model for distal gastrectomy when treating gastric cancer on the basis of data from 33,917 Japanese patients collected using a nationwide webbased data entry system. Ann Surg 262:295-303

9. Watanabe M, Miyata H, Gotoh M et al (2014) Total gastrectomy risk model: data from 20,011 Japanese patients in a nationwide internet-based database. Ann Surg 260:1034-1039

10. Dindo D, Demartines N, Clavien PA (2004) Classification of surgical complications: a new proposal with evaluation in a cohort of 6336 patients and results of a survey. Ann Surg 240:205-213

11. Lee KG, Lee HJ, Yang JY et al (2014) Risk factors associated with complication following gastrectomy for gastric cancer: retrospective analysis of prospectively collected data based on the Clavien-Dindo system. J Gastrointest Surg 18:1269-1277

12. Silber JH, Rosenbaum PR, Trudeau ME et al (2005) Changes in prognosis after the first postoperative complication. Med Care 43:122-131

13. Joseph B, Zangbar B, Khalil M et al (2015) Factors associated with failure-to-rescue in patients undergoing trauma laparotomy. Surgery $158: 393-398$

14. Tu RH, Lin JX, Zheng CH et al (2017) Complications and failure to rescue following laparoscopic or open gastrectomy for gastric cancer: a propensity-matched analysis. Surg Endosc 31:2325-2337

15. Washington K (2010) 7th Edition of the AJCC cancer staging manual: stomach. Ann Surg Oncol 17:3077-3079

16. Charlson ME, Pompei P, Ales KL et al (1987) A new method of classifying prognostic comorbidity in longitudinal studies: development and validation. J Chronic Dis 40:373-383 
17. Waljee JF, Windisch S, Finks JF et al (2006) Classifying cause of death after cancer surgery. Surg Innov 13:274-279

18. Zimmerman JE, Kramer AA, McNair DS et al (2006) Acute physiology and chronic health evaluation (APACHE) IV: hospital mortality assessment for today's critically ill patients. Crit Care Med 34:1297-1310

19. Knaus WA, Wagner DP, Draper EA et al (1991) The APACHE III prognostic system: risk prediction of hospital mortality for critically III hospitalized adults. Chest 100:1619-1636

20. Moreno RP, Metnitz PG, Almeida E et al (2005) SAPS 3-From evaluation of the patient to evaluation of the intensive care unit. Part 2: development of a prognostic model for hospital mortality at ICU admission. Intensive Care Med 31:1345-1355

21. Vincent JL, Moreno R, Takala J et al (1996) The SOFA (sepsisrelated organ failure assessment) score to describe organ dysfunction/failure. Intensive Care Med 22:707-710

22. Hartgrink H, Van de Velde C, Putter H et al (2004) Extended lymph node dissection for gastric cancer: who may benefit? Final results of the randomized Dutch gastric cancer group trial. J Clin Oncol 22:2069-2077

23. Cuschieri A, Joypaul V, Fayers P et al (1996) Postoperative morbidity and mortality after D1 and D2 resections for gastric cancer: preliminary results of the MRC randomised controlled surgical trial. Lancet 347:995-999

24. Hu Y, Huang C, Sun Y et al (2016) Morbidity and mortality of laparoscopic versus open D2 distal gastrectomy for advanced gastric cancer: a randomized controlled trial. J Clin Oncol 34:1350-1357

25. Katai H, Mizusawa J, Katayama H et al (2017) Short-term surgical outcomes from a phase III study of laparoscopy-assisted versus open distal gastrectomy with nodal dissection for clinical stage IA/IB gastric cancer: Japan Clinical Oncology Group Study JCOG0912. Gastric Cancer 20:699-708

26. Deitch EA (1992) Multiple organ failure. Pathophysiology and potential future therapy. Ann Surg 216:117-134

27. Jeong SH, Ahn HS, Yoo MW et al (2010) Increased morbidity rates in patients with heart disease or chronic liver disease following radical gastric surgery. J Surg Oncol 101:200-204

28. Jeong SH, Kim YW, Yu W et al (2015) High morbidity in myocardial infarction and heart failure patients after gastric cancer surgery. World J Gastroenterol 21:6631-6638

29. Gerestein C, Damhuis R, de Vries M et al (2009) Causes of postoperative mortality after surgery for ovarian cancer. Eur $\mathrm{J}$ Cancer 45:2799-2803

30. Bartlett EK, Roses RE, Kelz RR et al (2014) Morbidity and mortality after total gastrectomy for gastric malignancy using the American College of Surgeons National Surgical Quality Improvement Program database. Surgery 156:298-304

31. Zaydfudim V, Stukenborg G (2018) Effects of patient factors on inpatient mortality after complex liver, pancreatic and gastric resections. BJS Open 1:191-201

32. Ferraris VA, Bolanos M, Martin JT et al (2014) Identification of patients with postoperative complications who are at risk for failure to rescue. JAMA Surg 149:1103-1108

33. Sheetz KH, Krell RW, Englesbe MJ et al (2014) The importance of the first complication: understanding failure to rescue after emergent surgery in the elderly. J Am Coll Surg 219:365-370

34. Keegan MT, Gajic O, Afessa B (2011) Severity of illness scoring systems in the intensive care unit. Crit Care Med 39:163-169

Publisher's Note Springer Nature remains neutral with regard to jurisdictional claims in published maps and institutional affiliations. 